\section{THE SELECTIVE SIGMA-1 RECEPTOR AGONIST PRE-084 REDUCES NDMAR- MEDIATED EXCITOTOXIC BRAIN INJURY IN NEWBORN MICE}

M. Groß ${ }^{1}$, K. Medek ${ }^{1}$, M. Urbanek ${ }^{1}$, U. KiechlKohlendorfer $^{1}$, M. Keller ${ }^{2}$, E. Griesmaier ${ }^{1}$

${ }^{1}$ Department of Pediatrics IV, Medical University of Innsbruck, Innsbruck, Austria, ${ }^{2}$ Department of Pediatrics I, Neonatology, University Hospital of Essen, Essen, Germany

Background and aims: We have recently shown that Dextromethorphan is neuroprotective against excitotoxic and hyperoxic-induced brain injury. Beside its antagonistic effect on the NMDA [N-methyl-D-aspartate] receptor, DM also acts on sigma $(\sigma)$ receptors. Sigma agonists have been shown to be neuroprotective in several adult animal models of brain injury. Neuroprotection by sigma agonists is accomplished by a variety of mechanisms like inhibition of presynaptic glutamate release and attenuation of postsynaptic glutamate-evoked $\mathrm{Ca} 2+$ influx. The selective $\sigma 1$ receptor agonist Pre-084 [2-(4-Morpholinethyl) 1-phenylcyclohexanecarboxylate hydrochloride] has been shown to be neuroprotective in in-vitro and in-vivo studies of adult brain injury. The aim of this study was to evaluate the effect of Pre-084 in NMDAR-mediated excitotoxic brain injury in newborn mice.

Methods: 5-day-old mice pups were injected intracranially with ibotenate, a glutamate analogon. 1 hour after injury pups were randomly injected intraperitoneally (i.p.) with i) $0.1 \mu \mathrm{g} / \mathrm{g}$ body weight (bw) ii) $10 \mu \mathrm{g} / \mathrm{g}$ bw iii) PBS as control. Enpoints were set at postnatal day 6 and 10 and processed for histological analysis.

Results: Pre-084 reduced NMDAR-mediated excitotoxic brain injury in grey matter if administered 1 hour after injury. The low dose of $0.1 \mu \mathrm{g} / \mathrm{g}$ body weight was as effective as the high dose of $10 \mu \mathrm{g} / \mathrm{g}$ bw compared to PBS injected control animals.

Conclusion: We show for the fist time a protective effect of the selective $\sigma 1$ receptor agonist Pre-084 in an animal model of neonatal excitotoxic brain injury. Sigma agonists show a great protective potential and further analysis on the underlying mechanisms are ongoing.

\section{WIDESPREAD WHITE MATTER DAMAGE IS SEEN ON DTI IN YOUNG ADULTS BORN PRETERM WITH VERY LOW BIRTH WEIGHT (VLBW)}

L. Eikenes ${ }^{1}$, G.C. Løhaugen ${ }^{2,3}$, A.-M. Brubakk ${ }^{3,4}$, J. Skranes ${ }^{2,3,4}$, A. Håberg ${ }^{1,5,6}$

${ }^{1}$ Dept of Circulation and Medical Imaging, Norwegian University of Science and Technology, Trondheim, '2Dept of Pediatrics, Sørlandet Hospital, Arendal, ${ }^{3}$ Dept of Lab Medicine, Children's and Women's Health, Norwegian University of Science and Technology, ${ }^{4}$ Dept of Pediatrics, St Olav's

University Hospital, ${ }^{5}$ Dept of Neuroscience, Norwegian University of Science and Technology, ${ }^{6}$ Dept of Diagnostic Imaging, MR Center, St Olav's University Hospital, Trondheim, Norway

Background and aims: Perinatal brain injury in very low birth weight (VLBW) preterms is associated with permanent changes in white matter integrity and connectivity, and to neurodevelopmental problems including cognitive deficits.

The aim of study was to investigate whether being born with VLBW leads to white matter damage that persist into adulthood, and to examine the relationship between white matter integrity and perinatal data and IQ in the VLBW group.

Methods: Forty-nine VLBW young adults and 59 term controls were scanned at 1.5 T at ages 18-22 with DTI. Voxelwise maps of fractional anisotropy (FA) were calculated and Tract-Based Spatial Statistics was carried out to test for voxelwise differences between groups. Cognitive function was assessed with WAIS-III. The relationships between $F A$ and total $I Q$ and perinatal variables were explored.

Results: In VLBW adults all major central and posterior white matter tracts had reduced FA, mainly caused by an increase in the two lowest eigenvalues. FA correlated positively with birth weight and negatively with number of days on mechanical ventilator and in NICU. More than half of the VLBW subjects obtained a subnormal IQ score. The FA-IQ correlation analyses demonstrated positive correlations between $F A$ and IQ in regions including corpus callosum and long and short association tracts.

Conclusions: Preterm birth with VLBW results in long-term irreversible changes in white matter 
microstructure that relate to inferior IQ in VLBW young adults. Lower birth weight and perinatal problems seem to have permanent negative effects on white matter integrity.

\section{7}

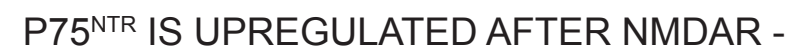
MEDIATED EXCITOTOXIC BRAIN INJURY IN NEWBORN MICE

\section{K. Wegleiter ${ }^{1}$, E. Griesmaier ${ }^{1}$, G. Schlager ${ }^{1}$, M. Urbanek ${ }^{1}$, M. Keller ${ }^{2}$ \\ ${ }^{1}$ Department of Paediatrics IV, Neonatology, Neuropaediatrics and Metabolic Diseases, Medical University Innsbruck, Innsbruck, Austria, \\ ${ }^{2}$ Department of Paediatrics, Neonatology, \\ University Hospital Essen, Essen, Germany}

Background and aims: Perinatal brain injury, leading to lifelong neurological handicaps, depicts a major problem in preterm infants. It has been shown that activation of $\mathrm{P} 75$ neurotrophin receptor $\left(\mathrm{P} 75^{\mathrm{NTR}}\right)$ plays a role in hypoxic-ischaemic and inflammation-mediated brain injury in adults. The role of P75 ${ }^{\mathrm{NTR}}$ in the pathogenesis of perinatal brain injury is unknown. We hypothesized that activation of the NMDA (N-methyl-D-aspartate) receptor in the immature brain induces an upregulation of P75 ${ }^{\text {NTR }}$ and knock out of P75 ${ }^{\text {NTR }}$ decreases brain injury.

Methods: We subjected 5-day-old P75 ${ }^{\text {NTR }}$ knock out (KO) and wild type (WT) mice to excitotoxic brain injury by a single intracranial ibotenate (glutamate analogon) injection. P75 ${ }^{\mathrm{NTR}}$ expression was analysed at 4,8 and 24 hours after brain injury by immunohistochemical staining for p75 NTR positive cells. Furthermore the number of activated caspase- 3 positive cells was evaluated 24 hours after brain injury in P75 ${ }^{\mathrm{NTR}} \mathrm{KO}$ and WT animals.

Results: In the WT cohort excitotoxicity significantly increased the number of $\mathrm{P} 75^{\mathrm{NTR}}$ positive cells in white (WM) and grey matter (GM) at 4 hours (GM $p<0,01$; WM $p<0,01)$ and in WM also at $8(p<0,01)$ and $24(p<0,05)$ hours after injury. The number of caspase-3 positive cells was significantly decreased in WM in KO compared to WT animals $(p<0.05) 24$ hours after injury.

Conclusion: We show the potential role of P75 NTR in a neonatal animal model of perinatal brain injury. Studies analysing detailed interactions of P75 ${ }^{\text {NTR }}$ in developmental brain injury are ongoing.
198

\section{MOLECULAR CHANGES AFTER WHOLE BODY COOLING ON PIGLETS WITH INDUCED TRANSIENT HYPOXIC-ISCHEMIA IN THE DEVELOPING BRAIN}

L. Olson', S. Faulkner², K. Lundströmer ${ }^{3}$, N.J. Robertson ${ }^{2}$, H. Lagercrantz ${ }^{1}$, U. Åden ${ }^{1}$, L. Olson ${ }^{3}$, D. Galter ${ }^{3}$

${ }^{1}$ Department for Women and Childrens Health, Karolinska Institutet, Stockholm, Sweden, ${ }^{2}$ Institute for Women's Health, UCL Medical School, London, London, UK, ${ }^{3}$ Department of Neuroscience, Karolinska Institutet, Stockholm, Sweden

Objective: To study the effect of whole body cooling and/or xenon treatment on molecular changes induced by transient hypoxic-ischemia $(\mathrm{HI})$ in the developing brain.

Methods: Experiments were performed under UK Home Office licence in accordance with UK guidelines. Newborn Large-White piglets were anesthetized and subjected to controlled transient HI. Piglets remained anesthetized and randomised to: (i) no treatment (ii) whole body cooling to a rectal temperature of $33.5 \pm 0.5{ }^{\circ} \mathrm{C}$ after $\mathrm{HI}$; (iii) xenon or (iv) cooling + xenon treatment. (v) naïve group subjected only to anaesthesia. Piglets were subjected to intensive care and monitoring. After euthenasia brains were perfused with $4 \%$ formalin and processed for in situ hybridization and immunohistochemistry. To characterize possible effects $\mathrm{HI}$ and treatments on the transcriptional activity of key neuronal and glial genes, we used in situ hybridization with probes designed to detect mRNA encoding HSP70, MAP2 and GFAP.

Results: HI caused GFAP mRNA levels to increase in cortex cerebri, but not in striatum. None of the treatments could counter act the increase in cortex. HSP70 mRNA was increased by $\mathrm{HI}$ in cortex and striatum. Treatments enhanced cortical increases, while slightly counteracting striatal. MAP2 mRNA decreased in cortex by HI. Protective effects were noted by both xenon and hypothermia, although not additive. Striatal levels were lower then cortical, although the general pattern was similar to cortex. Understanding of the molecular mechanisms of hypothermic and other neuroprotective agents will assist in determining optimal combination(s) of neuroprotective agents for protection of the challenged newborn brain. 